\title{
El futuro del sistema agroalimentario de la Región Metropolitana de Buenos Aires: un ejercicio de prospectiva territorial
}

\section{The future of the agri-food system of the Metropolitan Region of Buenos Aires: a territorial prospective exercise}

Lisandro Esteban Martínez

Licenciado en Antropología. Investigador del Centro de Investigación en Economía y Prospectiva (CIEP). Instituto Nacional de Tecnología Agropecuaria (INTA). Av. Cerviño 3101 P.B. (C.P. 1425) Ciudad de Buenos Aires, Buenos Aires, Argentina, martinez.lisandroe@inta.gob.ar

Recibido: 4 de octubre 2018 || Aprobado: 13 de abril 2020

Resumen

Un equipo de profesionales pertenecientes al Instituto Nacional de Tecnología Agropecuaria (INTA), realizamos un ejercicio exploratorio de prospectiva territorial para la Región Metropolitana de Buenos Aires (RMBA) con foco en la producción y consumo de alimentos y el territorio metropolitano donde estos se enclavan. En lo que se definió como un sistema agroalimentario metropolitano (SAM), se construyeron posibles escenarios futuros para el mismo. Contando el INTA con ya más de una década de abordaje integral de la agricultura urbana y periurbana en la región, el conocimiento de la masa crítica de profesionales que allí trabajan nos permitió avanzar en la caracterización de la dinámica de un sistema altamente complejo. Estudios prospectivos de este tipo, donde se construyen y estilizan escenarios futuros plausibles, resultan un insumo de gran importancia para el diseño y la definición de políticas públicas.

Palabras clave: Prospectiva territorial; Sistemas agroalimentarios; Agricultura urbana y periurbana; Políticas públicas

Abstract

A team of professionals belonging to the National Institute of Agricultural Technology (INTA), carried out an exploratory exercise of territorial prospective for the Metropolitan Region of Buenos Aires (RMBA) with a focus on food production and consumption and the metropolitan territory where they are placed. In what was defined as a metropolitan agrifood system (SAM), possible future scenarios were built for it. Counting INTA with more than a decade of comprehensive approach to urban and peri-urban agriculture in the region, knowledge of the critical mass of professionals who work there allowed us to advance in the characterization of the dynamics of a highly complex system. Prospective studies of this type, where plausible future scenarios are constructed and stylized, are an input of great importance for the design and definition of public policies.

Key words: Territorial prospective; Agri-food systems; Urban and peri-urban agriculture; Public policies

Cita sugerida: Martínez, L.E. (2020). El futuro del sistema agroalimentario de la Región Metropolitana de Buenos Aires: un ejercicio de prospectiva territorial. Estudios Socioterritoriales. Revista de Geografía, (27), 045. DOI: https://doi.org/10.37838/unicen/est.27-045 


\section{INTRODUCCIÓN}

El objetivo de poder pensar en el largo plazo para un sistema agroalimentario metropolitano -SAM- (Sali et al., 2014) 1 como el que representa la Región Metropolitana de Buenos Aires (RMBA), hizo a la realización de un ejercicio prospectivo $^{2}$. El mismo fue llevado adelante por un equipo interdisciplinario de profesionales del INTA, conformado por investigadores y técnicos de la Estación Experimental Agropecuaria AMBA (EEA AMBA) y sus agencias de extensión rural (AERs), con amplia trayectoria en el estudio e intervención de las dinámicas agro-productivas y socio-técnicas de la región. Estos fueron asistidos en metodologías de prospectiva territorial ${ }^{3}$ por investigadores del hoy Centro de Investigación en Economía y Prospectiva $(\mathrm{CIEP})^{4}$, también dependiente del INTA. La estructura del artículo está dividida en tres partes. Primero, desarrollamos algunos aspectos del estado actual de debate en relación a la agricultura urbana y periurbana y a los sistemas agroalimentarios metropolitanos en el contexto global. Segundo, caracterizamos a la RMBA conceptualmente como un sistema agroalimentario metropolitano. Y tercero, damos cuenta del ejercicio que desarrollamos y de los escenarios a futuro que se construyeron. Haremos especial mención al valor de la prospectiva territorial como forma de abordaje oportuna para una más coherente, comprensiva y efectiva

1 "Desde un punto de vista espacial, el SAM representa la ciudad, o una conurbación, y la tierra que la rodea necesaria para la producción de alimentos. El SAM es entonces un sistema agroalimentario caracterizado por un alto nivel de complejidad en términos de actores involucrados, logística y gran cantidad y variedad de productos procesados" (Sali et al., 2014, p. 9) (traducción nuestra).

2 Por ejercicio prospectivo entendemos el proceso sistemático y participativo para construir visiones a medio y largo plazo, con el objetivo de orientar las decisiones que han de tomarse en el presente y movilizar acciones conjuntas para construir el futuro deseado (Vitale, Pascale Medina, Barrientos y Papagno, 2016).

3 La prospectiva territorial es un proceso global (y no sectorial), y se refiere al conjunto del territorio físico y humano que constituye el espacio en estudio (Vitale et al., 2016).

4 El INTA sufrió una serie de cambios de estructura, en donde se disolvió el Instituto de Investigación en Prospectiva y Políticas Públicas, quedando integrados sus equipos de trabajo al hoy CIEP. manera de indagar en el largo plazo para sistemas territoriales como el que se presenta.

\section{LA RELACIÓN CAMPO-CIUDAD Y LA SIGNIFICANCIA DE} LOS SISTEMAS AGROALIMENTARIOS METROPOLITANOS

Existe un renovado y marcado interés por entender y planificar los espacios de transición entre la ciudad y el campo, denominados generalmente como espacios periurbanos. Sin embargo, la comprensión y caracterización de estos espacios de interfaz parecen escapar a definiciones unívocas (Tacoli, 1998; Barsky, 2005). Que los entornos de las ciudades sean equiparables en distintos contextos dependerá necesariamente de las características de los mismos $^{5}$. Entre los varios aspectos que nos permiten relacionarlos existe uno que merece especial atención, esto es, su función en la producción de alimentos frescos para el abastecimiento urbano. La visibilización y revalorización de estas producciones en las cercanías o dentro de las ciudades ha logrado instalarse en la agenda pública internacional desde hace ya algunas décadas (Smit y Nasr, 1992; Sommers y Smit, 1994; Mougeot, 2000; Bakker, Dubbeling, Gündel, Sabel-Koschella y de Zeeuw, 2000). Estas, en un mundo que tiende día a día hacia mayores tasas de urbanización, parecen tornarse en un aspecto central para la sostenibilidad de las ciudades y, con más razón aún, de las regiones metropolitanas (Satterthwaite, McGranahan y Tacoli, 2010). Aunque este tipo de sistemas ha existido desde siempre (por lo menos desde la revolución neolítica), siendo parte constitutiva de los procesos de crecimiento urbano, más recientemente han sido revalorizados por organismos como la ONU y $\mathrm{FAO}^{6}$ insistiendo en la

5 Un aspecto diferenciador básico de los espacios periurbanos es determinar sobre qué tipo de ambiente natural se enclava la ciudad y su región. Las diferencias en tipo y disponibilidad de recursos naturales, como suelo, agua y biodiversidad, junto con variaciones climáticas diferenciales, hacen que necesariamente los espacios periurbanos deban pensarse y compararse en base a estas diferencias (Sali et al., 2014).

6 Estos han sido incluidos como parte de las políticas estratégicas del Pacto de Política Alimentaria Urbana de Milán (MUFPP) de 2015, así como en la Nueva Agenda Urbana HABITAT III de la UN de 2016. 
necesidad de consolidar y potenciar el rol de la llamada agricultura urbana y periurbana (AUP) ${ }^{7}$ para lograr ciudades más sostenibles y resilientes (Smit, Ratta y Nasr, 2001; van Veenhuizen, 2006; van Veenhuizen y Danso, 2007; De Zeeuw, van Veenhuizen y Dubbeling, 2011), destacando a su vez dentro de esta, el rol de la agricultura de base familiar (Boukharaeva y Marloie, 2006; Parés, 2009; Feito, 2017).

Desde una perspectiva amplia, centrada en los aportes de la AUP para un desarrollo más sostenible (en sus tres dimensiones, tanto social como económica y ambiental), se destaca el rol que la misma juega en relación a la seguridad alimentaria, la generación de empleos, el reciclado de nutrientes, la generación de servicios eco-sistémicos, todos aspectos que nos muestran la multifuncionalidad de este tipo de agricultura (van Veenhuizen, 2006; Aubry et al., 2012). Al mismo tiempo, y en contraposición, mostrando los problemas que puede acarrear la producción de cercanía, producir alimentos en suelos contaminados, el riesgo de irrigar con aguas poluídas, junto a deficiencias en los sistemas productivos con condiciones socio-laborales precarias y un mal uso de productos agroquímicos, pueden generar problemas sociales, de salud, ambientales y/o de inocuidad tanto para productores como para consumidores (van Veenhuizen, 2006).

Aun teniendo presente estos últimos condicionantes, los sistemas de cercanía representan formas que acarrean más beneficios que negatividades, algo que ha sido mundialmente reconocido, como ya expresamos. Uno de sus principales beneficios reside en que permiten disminuir el costo del trasporte tanto como su

7 Podemos definir a la AUP como "una producción ubicada dentro (intra-urbana) o en la periferia (peri-urbana) de un pueblo, una ciudad o una metrópolis que cultiva y cría; procesa y distribuye una diversidad de productos alimentarios y no alimentarios, (re)utilizando en gran medida recursos humanos $y$ materiales, productos y servicios que se encuentran en y alrededor de dicha zona urbana, y a su vez provee recursos humanos y materiales, productos y servicios en gran parte para esa misma zona urbana" (Mougeot, 2000, p. 10) (traducción nuestra). Existen diferencias entre la agricultura netamente urbana y la que se encuentra en la periferia (periurbana) (Opitz, Berges, Piorr y Krikser, 2016), sin embargo aquí no desarrollaremos esas diferencias. impacto ambiental (huella de carbono), sobre todo para alimentos frescos donde, además, el tiempo entre la cosecha y consumo implica pérdidas de cualidades y eventualmente la generación de desperdicios (otro aspecto que ha ganado especial atención en términos de sostenibilidad). Sumado a esto, sistemas de comercialización de mayor intermediación redundan en mayores costos, aumento del precio de los alimentos y en menores oportunidades de capitalización para los productores. Por todo esto, promover canales cortos de comercialización, como parte importante de circuitos cortos de abastecimiento, representa una manera de apuntalar y promover las producciones de cercanía y su aporte a la sostenibilidad de las ciudades (Jarosz, 2008; Guiomar, 2011; Aubry y Kebir, 2013; Palacios-Argüello, Morganti y Gonzalez-Feliu, 2017; Dubois, 2018; Grando, Carey, Hegger, Jahrl y Ortolani, 2018), así como un empoderamiento de la comunidad que se afianza en una relación más directa entre productores y consumidores (Reed et al., 2018).

Todos estos aspectos se tornan mucho más pertinentes y determinantes para regiones metropolitanas como la RMBA, con más de quince millones de habitantes que necesitan un abastecimiento constante de alimentos. La dimensión y complejidad que adquiere la producción y el abastecimiento para áreas tan densamente pobladas hace a definirlas como sistemas agroalimentarios metropolitanos -SAM- (Sali et al., 2014). Estos implican tanto la producción de alimentos, fibras y/o bioproductos de distinto tipo en la región, junto con sus mecanismos de agregado de valor (agroindustrias) y comercialización (desde mercados, sobre todo locales aunque no solo estos, pasando por grandes cadenas de supermercados, a ferias y otras formas más directas de relación productor-consumidor), así como los hábitos y formas de consumo (tanto sea en el hogar, en locales de comidas elaboradas o por delivery) por parte de los residentes de las ciudades. Todo lo cual conforma un sistema altamente complejo. 
Entendemos por ello que pensar a las regiones metropolitanas como sistemas agroalimentarios debería ser algo prioritario dentro de la planificación en general. Este tipo de abordaje se encuadra dentro del concepto de "Urban Food Planning" (Morgan, 2009), donde se contempla la importancia de la producción y comercialización alimentaria de cercanía para la planificación urbanística. Es que los sistemas agroalimentarios (tanto sean estos locales, regionales, metropolitanos o, incluso, globales), dado su carácter multifuncional, tienen profundos efectos en vastos sectores de importancia para la planificación urbana (salud pública, justicia social, energía, agua, suelo y transporte, entre los más destacados).

Sin embargo, definir los límites de estos sistemas agroalimentarios metropolitanos resulta una tarea compleja. Es que, como sostuvimos, a la de por sí problemática definición del concepto de periurbano de forma precisa, subyace la de dar cuenta de estos como parte de la relación campo-ciudad. Quizás una manera de saldar en parte esta dificultad sea pensar en una bio-región urbana (Allen 2003), donde la ciudad se encuentra inserta en un territorio más basto, tensado en el trinomio rural-periurbano-urbano, siendo justamente el periurbano la interfaz de conexión y tensión hacia ambos lados, entre uno y otro de los ambientes más definidos en sus características intrínsecas (rural y urbano). Desde este enfoque, la planificación de un territorio debe contemplar la relación metabólica de la ciudad con su entorno (Díaz Álvarez, 2014). Así, su impacto ambiental en términos de consumo de recursos y generación de residuos y efluentes, puede medirse como su huella ecológica (Pengue, 2017), entendida a su vez como una relación metabólica de vinculación sociedad-naturaleza.

En relación específica a los SAM, los mismos, como parte de las bio-regiones en que se enclavan, son impactados por el crecimiento urbano en un proceso de geofagia (Pengue, 2017), donde las ciudades funcionan como 'depredadoras' de suelo productivo (Colantoni, Grigo- riadis, Sateriano, Venanzoni y Salvati, 2016). Este modelo depredador, que no contempla el valor intrínseco del suelo agrícola para la producción de alimentos, movilizado por la valorización inmobiliaria especulativa, transforma los establecimientos rurales y periurbanos en suelo vacante $y$, a la larga, en un espacio degradado (Garay, 2003). Esta falta de valorización del suelo productivo, que al perderse resulta casi imposible recuperar, representa un costo oculto del llamado desarrollo inmobiliario y urbano que, entendemos, es necesario contemplar si lo que se quiere es lograr ciudades más sostenibles.

Por estos motivos, pensar las necesidades alimentarias de una gran metrópolis, su huella ecológica, los impactos y demandas de recursos sobre su bio-región, la disponibilidad de tierra y agua en sus cercanías, nos hacen revalorizar las particularidades y potencialidades de las zonas de interfaz, esto es, los periurbanos y su rol en la producción de alimentos, junto con la sostenibilidad de todo el sistema agroalimentario metropolitano, lo que obliga la necesaria planificación de estos territorios (Verdaguer Viana-Cárdenas, 2010). Teniendo todo esto presente, haremos ahora foco en la RMBA definiéndola entonces como un sistema agroalimentario metropolitano.

\section{El SISTEMA AGROALIMENTARIO DE LA RMBA}

Existen dos motivos de peso para definir a la RMBA como un sistema agroalimentario metropolitano. El primero, de carácter histórico (como ocurre generalmente con todas las ciudades), nos habla del desarrollo de la metrópolis y su vinculación con su entorno periurbano y bio-región en términos de producción y provisión de alimentos (así como de otro tipo de bio-productos), lo que fuera y continua siendo una relación fuerte que promoviera el desarrollo de producciones e industrias como la láctea, la cárnica, la hortícola o la florícola, entre otras. A su vez, y de mayor relevancia para un estudio prospectivo, observamos la situación actual de las producciones de cercanía y su rol en términos de seguridad alimentaria, máxime para el abastecimiento de los más de quince millones 
de personas que habitan la región (además de abastecer a otras regiones).

Pero sumado a esto, como sostuvimos, la multifuncionalidad de la AUP aporta muchas más funciones a la RMBA reforzando su definición como un SAM (generación de empleos, servicios eco-sistémicos, reciclado de desperdicios, resguardo de áreas verdes y contención del crecimiento urbano descontrolado, etc.). Sin embargo, la misma dificultad por delimitar con precisión qué se entiende por RMBA, como una entidad definida, implica dificultades para definirla a su vez como un SAM.

Siendo un territorio sumamente basto y diverso, signado por un proceso de metropolización que, como en todo proceso similar, genera un crecimiento urbano acelerado y una complejización creciente del territorio, implica la inherente dificultad para definir con precisión los límites de dicha expansión. Pero sumado a esto, la RMBA adquiere mayores dificultades de definición producto de nuclear en su interior a la que es la ciudad capital de la Argentina, rodeada por municipios interrelacionados que en conjunto representan la mayor área urbana del país, un entramado político y administrativo yuxtapuesto, contrastante, conflictivo $y$, por las mismas razones, resbaladizo en términos conceptuales.

Si bien no existe una única definición que encuadre la metropolización de Buenos Aires, coexistiendo nombres y recortes que se superponen e implican mutuamente (como Gran Buenos Aires, conurbano bonaerense, área metropolitana), aquí tomamos la noción de región metropolitana para englobar a esta gran metrópolis. Esta se compone de la Ciudad Autónoma de Buenos Aires (CABA), núcleo de la región con tres millones de habitantes y que, por obra de procesos históricos y simbólicos suele pensarse como algo distinto y separado del resto de la conurbación ${ }^{8}$, rodeada por cuarenta partidos

8 La entidad de la CABA y su vinculación con el resto de la conurbación ha sido una relación conflictiva (desde su nacionalización en 1880 hasta la obtención de su autonomía en 1996) que, en gran medida, ha escindido a la misma y obturando la necesaria planificación del territorio como un todo (Gorelik, 2015). de la provincia de Buenos Aires ${ }^{9}$, que en total suman hoy más de quince millones de habitantes. Muchos de estos partidos circundantes, los que conforman las llamadas tercera y cuarta coronas, conservan aún gran parte de sus tierras como tierras rurales, razón por la cual representan un espacio de suma importancia para plantear políticas públicas orientadas a la AUP de proximidad (Barsky, 2007). Mientras tanto, el área estrictamente urbana supone un área mucho más reducida ${ }^{10}$.

Más allá de estas dificultades, uno de los resultados conceptuales del ejercicio realizado fue la necesidad de definir la RMBA como un SAM (Kozel, Martínez, Taraborrelli y Carvalho, 2017). Tanto para comprender la funcionalidad en el abastecimiento (en términos de comercialización, logística, suministro, acceso, etc.) de forma diaria para millones de personas, como sobre todo por la significancia y aporte de las producciones de cercanía. Subrayando con ello su utilidad para comprender y dimensionar las problemáticas alimentarias de una gran región metropolitana como lo es la RMBA. Las implicancias en términos de problemas de acceso, de nutrición, la generación de crisis alimentarias en periodos de alta inflación monetaria, en la región más densamente poblada del país, con millones

9 Aquí tomamos la definición de RMBA dada por el Ministerio de Infraestructura, Vivienda y Servicios Públicos del Gobierno de la Provincia de Buenos Aires de su libro "Lineamientos Estratégicos para la Región Metropolitana de Buenos Aires. Plan Estratégico Territorial" (2007). Corresponde a los 40 partidos más próximos a Capital Federal junto con esta, según la lista que sigue: Almirante Brown, Avellaneda, Berazategui, Berisso, Campana, Cañuelas, Coronel Brandsen, Ensenada, Escobar, Esteban Echeverría, Ezeiza, Exaltación de la Cruz, Florencio Varela, Gral. Las Heras, Gral. Rodríguez, Gral. San Martín, Hurlingham, Ituzaingó, José C. Paz, La Matanza, La Plata, Lanús, Lomas de Zamora, Luján, Marcos Paz, Malvinas Argentinas, Merlo, Moreno, Morón, Pilar, Presidente Perón, Quilmes, San Fernando, San Isidro, San Vicente, San Miguel, Tigre, Tres de Febrero, Vicente López y Zárate.

10 Frente a los aproximadamente $13.000 \mathrm{~km} 2$ de la RMBA, su área urbana abarca solo $2.440 \mathrm{~km} 2$. Este último dato implica que aproximadamente un tercio de la población nacional vive en una superficie que representa menos del $0,1 \%$ del territorio continental argentino, lo que podríamos caracterizar como la "macrocefalia porteña" o, para utilizar otra metáfora, la "cabeza de Goliat" argentina como la nombrara Ezequiel Martínez Estrada en 1940. 
de personas con problemas de empleo, de pobreza, de acceso a recursos, etc. (Aguirre, 2005), no hacen más que dar mayor significancia a un abordaje sistémico de los problemas alimentarios que aquí se presentan. $Y$ sin desestimar tampoco la importancia de la región en la puja política a nivel tanto provincial como nacional.

\section{CARACTERIZACIÓN DE LA PRODUCCIÓN AGROALIMENTARIA DE LA $R M B A$}

Una de las principales dificultades para una cabal apreciación de la importancia de la AUP en el SAM bonaerense es la falta de datos actualizados. La cantidad y características de los productores agropecuarios que residen en la región no pueden determinarse de forma precisa. Aun así, a través de distintas fuentes y del propio desarrollo del ejercicio, pudimos dimensionar la significancia de los mismos para la región. Así, podemos hablar de que entre tres mil y cuatro mil explotaciones agropecuarias residen en las cercanías $^{11}$ (Ministerio de Infraestructura, Vivienda y Servicios Públicos, 2007; Lipori, Cad, Palacios y Barrionuevo, 2011; INTA, 2012; Barsky, 2013; Kozel et al., 2017). Alrededor de 1.500 de estas corresponden a agricultores familiares de producciones intensivas hortícolas (Benencia y Quaranta 2005), en escalas de producción pequeñas de entre una y cuatro hectáreas. Este tipo de producción, característica de la región y que representa uno de los principales polos hortícolas de la Argentina, ha sido desarrollada históricamente por migrantes. Desde fines del siglo XIX y gran parte del siglo XX estos provinieron de ultramar (portugueses, italianos y españoles),

11 El último Censo Nacional Agropecuario con datos consistentes fue el realizado en el año 2002. El siguiente, del año 2008, tuvo problemas de implementación (condicionado por el llamado "conflicto con el Campo") con lo cual no brindó datos consistentes. Sumado a estos, el Censo Hortiflorícola bonaerense del año 2005 sirve para brindar más datos, pero que ante un proceso que es sumamente dinámico y cambiante, no hace más que resaltar la falta de datos actualizados. Aunque la tendencia en la cantidad de explotaciones ha sido a la baja (Lipori et al., 2011), el fuerte dinamismo del sector agropecuario metropolitano no permite reafirmar que esta tendencia se haya prolongado necesariamente. La realización de un nuevo Censo Nacional Agropecuario en 2018, cuyos resultados aún son preliminares, puede subsanar estas deficiencias. pero desde la década de 1970 viene siendo desarrollada cada vez más por migrantes de origen boliviano, una de las colectividades que más ha avanzado en ocupar estos espacios productivos (Garcia y Le Gall, 2009). Esta es una de las producciones que más fuertemente aporta a la seguridad alimentaria metropolitana, abasteciendo gran parte de las verduras frescas que se consumen en la región, tanto de hoja como de fruto ${ }^{12}$.

El destacado peso de la horticultura en el SAM hizo que durante el ejercicio, la misma tendiera a 'eclipsar' la dinámica y la funcionalidad del sistema en torno a dichas producciones. Sin embargo, si bien importante, no es el único tipo de producciones que existen en la región. De hecho, la multifuncionalidad de la AUP metropolitana se evidencia y gana significancia en una gran diversidad de producciones. Estas explotaciones corresponden a productores florícolas, porcinos, avícolas, tamberos, apicultores y de ganadería de cría e invernada, los cuales refuerzan la diversidad y multifuncionalidad que presenta la AUP en la región (Ministerio de Infraestructura, Vivienda y Servicios Públicos, 2007; Lipori et al., 2011; INTA, 2012; Barsky, 2013). Y si bien todos estos influyen de distintas maneras en el abastecimiento alimentario, algo sumamente relevante para la seguridad alimentaria regional, lo cierto es que este entramado de actores conforma solo la etapa de producción agroalimentaria del SAM bonaerense.

Así también, producción granaria extensiva (donde la escala de los predios es mayor) se presenta sobretodo en el borde externo de la región, introduciéndose de forma intersticial hacia dentro de las áreas periurbanas, siendo un frente de presión desde afuera que compite también (lo mismo que los usos urbanos de suelo) con la producción de pequeña escala periurbana que abastece el mercado interno (Giusti y Prividera, 2015).

Por todo esto, la diversidad de actores productivos refuerza la multifuncionalidad de la AUP

12 Entre el $60 \%$ y el $90 \%$ según las estaciones (Benencia, 2004), donde sobresalen el consumo de tomates, pimientos, berenjenas, la totalidad de verduras de hoja y crucíferas, así como algo de la fruta. 
para el SAM. Pero a la vez, lo que se evidencia es un sistema que desde la producción al consumo involucra muchos más actores. Para comenzar, la totalidad de personas vinculadas directamente con la AUP es mucho mayor que el número de explotaciones o productores (Benencia y Quaranta, 2005). Para el caso de la horticultura, una actividad intensiva en mano de obra, la cifra total de actores involucrados directamente puede rondar las 10 mil personas (contando productores, medieros, asalariados rurales, familiares, vendedores de insumos y de maquinaria, técnicos, etc.). Si sumamos al resto de las actividades productivas accedemos a un número aún mayor. Y si además contemplamos a todos los actores involucrados en el sistema de comercialización (desde los consignatarios que recolectan la producción en los predios, los actores de los distintos mercados concentradores, hasta la venta minorista y otras formas de comercialización) podemos comenzar a dimensionar un sistema altamente complejo que involucra a decenas de miles de personas. Por ello, el sistema agroalimentario metropolitano es algo mucho más vasto que, en última instancia, involucra a toda la población de la región, en relación a una actividad tan indispensable para el sostenimiento de la vida como lo es la alimentación.

Como dijimos, la zona hortícola de la región metropolitana representa uno de los polos de producciones intensivas más importantes del país. Si bien ha sufrido cambios en su morfología a lo largo de las últimas décadas, hoy en día se calcula que existen aproximadamente unas 17.000 hectáreas hortícolas sumadas a unas 1.200 hectáreas florícolas (Barsky, 2013). El crecimiento urbano ha afectado significativamente la distribución espacial de este tipo de producciones, desplazando a muchas de estas, sobre todo en la zona norte de la región metropolitana. Este proceso hizo que la actividad hortícola se haya desplazado hacia la zona sur, al Gran La Plata, donde se nuclean hoy la mayoría de las explotaciones. Esto ha sido motorizado por la incorporación de tecnología para aumentar la productividad por ha/año y volver más competitivos y rentables los establecimientos. El prin- cipal cambio ha sido la incorporación del invernáculo como forma de intensificación, lo que permite obtener más cosechas por año. El último dato de superficie bajo cubierta contabiliza que unas 5.461 ha, solo en la zona del Gran La Plata, Florencio Varela y Berazategui (Miranda, 2017), es decir un tercio del total.

Aparejados a la incorporación masiva del invernáculo se suman problemas ambientales por la mayor necesidad de productos químicos para el control de plagas, la impermeabilización del suelo y los problemas de control de excesos hídricos (García, 2011; Stupino, Frangi y Sarandón, 2012; Cuellas, 2015). De allí que en el desarrollo del ejercicio prospectivo se haya evidenciado este proceso como uno de los principales aspectos del impacto ambiental de la AUP en la región. Concomitantemente con ello, la misma es afectada por la presión del uso urbano del suelo, siendo uno de los aspectos más sensibles del ordenamiento territorial metropolitano. Lo cual, sumado a las situaciones de precariedad en la tenencia de la tierra, a condiciones socio-laborales de explotación y auto-explotación, denotan la condición de suma conflictividad y fragilidad de la AUP regional.

UN EJERCICIO DE PROSPECTIVA TERRITORIAL PARA EL SAM BONAERENSE

Hasta aquí hemos situado el debate en relación a los sistemas agroalimentarios de cercanía y metropolitanos, y caracterizado al SAM bonaerense. Si bien todo esto fue producto también del ejercicio realizado, decidimos comenzar primero por situar el debate, para ahora sí avanzar en describir cómo se encausó el desarrollo prospectivo propiamente dicho.

Debemos comenzar por aclarar que el ejercicio de prospectiva territorial que realizamos fue producto de un pedido institucional ${ }^{13}$. Por ello, es necesario embarcar el accionar del INTA en la

13 A raíz de una auditoria interna a la EEA AMBA, se determinó la necesidad de realizar un estudio prospectivo con escenarios, para poder visualizar cuáles eran los futuros posibles de los territorios sobre los que se busca incidir. Para el ejercicio se dispuso en principio de un tiempo no mayor a seis meses. 
región. Este organismo de ciencia y técnica del estado argentino trabaja desde hace más de sesenta años en pos de la generación de capacidades científicas y tecnológicas que dinamicen los sistemas de producción de alimentos, fibras y energías, aportando al desarrollo sostenible de los territorios en sus dimensiones ambientales, sociales y económicas. En este marco, la atención de los espacios periurbanos como un tipo de territorio que demanda un abordaje especial, ha generado políticas públicas específicas a partir de una institucionalidad acorde. Así, la creación de la Estación Experimental Agropecuaria AMBA en 2011 (junto con la integración de viejas y nuevas Agencias de Extensión Rural -AERen la región) coronó un proceso de maduración iniciado a mediados de la década del 2000, por el cual se da prioridad al abordaje de la AUP de base familiar en la región (Barsky y Aboitiz, 2012). Los ejes de intervención para la nueva experimental se enfocan tanto a los aspectos que hacen a la producción y comercialización (ejes tradicionales dentro del INTA), como a la promoción de la soberanía alimentaria (entendida como la capacidad de una sociedad de definir su propia política agroalimentaria), la equidad social, el cuidado del ambiente y el ordenamiento territorial (Barsky, 2013). A su vez, este abordaje se instrumenta a través de un enfoque territorial por medio de la conformación de cuatro grandes zonas, como son los territorios norte, sur, oeste y urbano. En este contexto, la necesidad de contar con una mirada de más largo plazo de los procesos sobre los que se busca incidir, y que nutra la toma de decisión en términos estratégicos, generó la demanda de un ejercicio de prospectiva territorial para este ámbito. Para ello, se recurrió al entonces Instituto de Investigación en Prospectiva y Políticas Públicas del INTA para coordinar el mismo, asistiendo en metodologías de prospectiva territorial.

\section{METODOLOGÍAS PROSPECTIVAS DE ABORDAJE TERRITORIAL}

Dejando en claro que el futuro es en gran medida incognoscible y que por la naturaleza misma de la realidad no puede ser abordado científicamente, no deja de ser cierto, por otro lado, que la necesidad de proyectarnos a futuro es una fuerte necesidad humana, no importa a qué grupo o época en particular nos refiramos. Contar con una proyección de hacia dónde nos dirigimos representa una necesidad imperiosa frente a la naturaleza incierta del porvenir. Tanto sea en sociedades ancladas en formas tradicionales (que parecen querer impedir el cambio) o, por el contrario, en aquellas más modernas en donde la velocidad de las transformaciones dificultan el pronóstico y la acción planificada, sea como fuera, la necesidad de plantear hojas de ruta frente a la incertidumbre inherente al porvenir hacen a la necesidad de pensar el futuro. Sin ahondar en cómo esta necesidad ha sido colmada desde distintas formas de pensamiento (sean míticas, religiosas, políticas o científicas), desde la segunda mitad del siglo XX se comprendió que, más allá de aceptar la naturaleza incierta del futuro, no se podía eludir la necesidad de pensar el mismo para orientar las acciones presentes. Así comienza a conformarse un conjunto de teorías y metodologías de los que luego se llamarían 'estudios del futuro' y en particular de la prospectiva. Esta disciplina ya cuenta con más de medio siglo de avances $y, \sin$ ahondar demasiado en su desarrollo, tomamos una definición (de las muchas posibles) para aclarar qué entendemos por prospectiva:

\footnotetext{
La prospectiva supone una reflexión estructurada y sistemática acerca de las alternativas futuras de un país, territorio, sector o institución, mediante la interacción organizada con expertos, redes y comunidades, basada en un diálogo fundamentado en hechos y datos. Implica la construcción de visiones de futuro estructuradas, verosímiles, innovadoras, transformadoras y con posibilidades de realización. (Medina Vásquez, Becerra y Castaño, 2014, p. 48)
}

Tomando esta definición, de lo que se trata entonces es de visualizar futuros posibles por medio de tallerizar con grupos de expertos y actores involucrados en torno a un objeto de estudio particular (que puede ser desde un país o una institución, a un sector o un territorio, como en nuestro caso). Los debates y conceptualizacio- 
nes sobre dicho objeto, y su propia delimitación y construcción, intentan por medio de definir los factores claves que hacen a su desenvolvimiento, pensar en el largo plazo del mismo de forma sistémica, con el objetivo principal de orientar la toma de decisiones en el presente. Por ello, el objetivo principal de la prospectiva no es predecir el futuro con más o menos acierto (lo cual resulta imposible), sino orientar y ayudar a la toma de decisiones en aspectos claves del desenvolvimiento de un sistema para alcanzar lo que, desde el presente, se visualiza como un futuro deseado. Una de las metodologías para visualizar ese futuro (o futuros) es construir de forma colectiva y consensuada escenarios prospectivos que permiten condensar los procesos que definen el devenir del objeto particular de que se trate, y que consiguen sistematizar las alternativas para alcanzar un futuro deseado, tanto como evitar uno indeseado. En nuestro caso, debido a que nuestro objeto de estudio, como desarrollamos en los apartados anteriores, es un objeto de índole territorial (el SAM bonaerense), entendiendo al mismo tanto físicamente como a la vez producto de relaciones humanas, la prospectiva desarrollada es por ello también de carácter territorial, expresión de un proceso global y no sectorial (Vitale et al., 2016).

\section{ETAPAS DEL EJERCICIO}

El diagramado del ejercicio consistió en una serie de talleres iterativos con los técnicos e investigadores de la EEA AMBA, a partir de los cuales se pudo realizar un diagnóstico y la caracterización de la RMBA como un SAM (resultado de lo cual dimos cuenta en los apartados anteriores). Se comenzó con una etapa previa de sensibilización de la terminología prospectiva y de sus herramientas y metodologías. Luego se conformó el grupo de trabajo que participaría de los talleres, integrado por unos veinte técnicos e investigadores representando a todos los territorios de la RMBA. Uno de los condicionantes en la realización del ejercicio fue que, al ser un ejercicio institucional, el sesgo 'intiano' no pudo ser evitado, dado que tanto el grupo que participó de los talleres como las salidas a campo y las consultas a expertos fueron protagonizadas por trabajadores y técnicos del INTA. $Y$ aunque conocimos $y$ entrevistamos a productores y otros actores del SAM, la impronta 'intiana' fue la que marcó gran parte del desarrollo del ejercicio. Con lo cual este sesgo no puede ser obviado, condicionando tanto el desarrollo como los resultados. Esto no implica quitarle valor al ejercicio, sino solamente tener presente desde la óptica de cuáles actores fue abordado.

El ejercicio se dividió en dos grandes etapas. En la primera, durante los meses de agosto a diciembre de 2016 y una vez conformado el grupo de trabajo, se tallerizó y se llegó rápidamente (para los tiempos en que habitualmente se desarrollan los ejercicios prospectivos) a la conformación de lo que llamamos proto-escenarios, con una base argumental de los procesos definitorios para el devenir del SAM. La segunda parte, desarrollada entre los meses de marzo a junio de 2017, consistió en poner a consideración de más interlocutores los resultados alcanzados, en donde a partir de los aportes recibidos, se redefinieron y profundizaron en aspectos que no habían sido tenidos en cuenta con el suficiente detalle.

Comenzando por describir la primera etapa, en total se desarrollaron cinco talleres grupales. En los primeros encuentros se definió el objeto de estudio, el foco y se comenzó a pensar en el horizonte temporal (que quedó definido en dos horizontes distintos, 2030 y 2050, como exponemos más adelante). Como expresamos previamente, el objeto de estudio quedó definido como el SAM bonaerense ${ }^{14} y$ el foco del mismo se refirió a la producción y consumo de alimentos y su relación con el territorio metropolitano donde estos se enclavan (relación alimentos-territorio). Luego se ordenó el debate y el abordaje del foco a través de definir escalas y dimensiones de análisis. A

14 En la caracterización del SAM tuvo especial importancia la historia de conformación de la Región Metropolitana de Buenos Aires, los intentos por planificar su crecimiento y su `efecto gravitatorio' sobre su entorno. 
partir de caracterizar el SAM y la AUP regional, de las salidas a campo, de las visitas a mercados concentradores y a productores (hortícolas, porcinos y florícolas), y de los intercambios $y$ tareas que se daban tanto dentro como fuera de los talleres, en los últimos se comenzaron a definir los factores críticos que hacen al desenvolvimiento del SAM (Cuadro 1).

Cuadro 1. Factores críticos por escala y dimensión

\begin{tabular}{|c|c|c|}
\hline Escala & Dimensión & Factores críticos \\
\hline \multirow{4}{*}{$\begin{array}{l}\text { Internacional / } \\
\text { Regional }\end{array}$} & \multirow{3}{*}{ Contexto geopolítico } & Regresión de la multipolaridad \\
\hline & & Aceleración del cambio tecnológico \\
\hline & & Dinámica regional sudamericana \\
\hline & Ambiental & Evolución del cambio climático \\
\hline \multirow{10}{*}{$\begin{array}{l}\text { Nacional / Pro- } \\
\text { vincial / Local }\end{array}$} & \multirow{2}{*}{ Económica } & $\begin{array}{l}\text { Valorización de la tierra y cambios en el uso de } \\
\text { suelo derivados del proceso de metropolización }\end{array}$ \\
\hline & & $\begin{array}{l}\text { Despliegue de actividades enmar- } \\
\text { cadas en la Bioeconomía }\end{array}$ \\
\hline & \multirow{6}{*}{ Político-institucional } & Proyecto de gobierno \\
\hline & & Política industrial y agroindustrial \\
\hline & & Política científico-tecnológica \\
\hline & & Política migratoria \\
\hline & & $\begin{array}{l}\text { Grado de consistencia de los marcos re- } \\
\text { gulatorios y jurisdiccionales }\end{array}$ \\
\hline & & $\begin{array}{l}\text { Políticas de ordenamiento y gober- } \\
\text { nabilidad de los territorios }\end{array}$ \\
\hline & \multirow[b]{2}{*}{ Socio-cultural } & Evolución de las pautas de consumo de alimentos \\
\hline & & $\begin{array}{l}\text { Visibilización y valorización so- } \\
\text { cial de la actividad hortícola }\end{array}$ \\
\hline \multirow{11}{*}{ Focal } & \multirow{8}{*}{ Socio-tecno-productiva } & $\begin{array}{l}\text { Evolución de la intensificación pro- } \\
\text { ductiva ("Invernaculización”) }\end{array}$ \\
\hline & & $\begin{array}{l}\text { Dinámica y pautas predominantes de la co- } \\
\text { mercialización de las producciones }\end{array}$ \\
\hline & & Parámetros de competitividad de las producciones \\
\hline & & $\begin{array}{l}\text { Evolución del actor productivo más di- } \\
\text { námico (“Bolivianización”) }\end{array}$ \\
\hline & & Políticas públicas específicas para la AUP \\
\hline & & $\begin{array}{l}\text { Disrupciones tecnológicas en la pro- } \\
\text { ducción de alimentos }\end{array}$ \\
\hline & & $\begin{array}{l}\text { Grado de conocimiento de prácticas apro- } \\
\text { piadas (BPA; "transición agroecológica") }\end{array}$ \\
\hline & & $\begin{array}{l}\text { Expansión y promoción de la agri- } \\
\text { cultura urbana (traspatio) }\end{array}$ \\
\hline & Socio-territorial & Conflictividad por el uso del suelo \\
\hline & \multirow{2}{*}{ Ambiental } & $\begin{array}{l}\text { Sostenibilidad ambiental y sanitaria compro- } \\
\text { metida (suelos, agua, manejo de residuos) }\end{array}$ \\
\hline & & $\begin{array}{l}\text { Variabilidad climática en un con- } \\
\text { texto de cambio climático }\end{array}$ \\
\hline
\end{tabular}

Fuente: elaboración personal 
Contando entonces con esta lista de factores críticos se realizó un ejercicio de importancia-incertidumbre, el cual permite trabajar la amplitud de apreciaciones sobre el futuro del grupo, llegando a una ponderación colectiva de los factores más importantes para el desenvolvimiento del foco. Así, los factores más importantes e inciertos son considerados incertidumbres críticas (en rojo en el cuadro), los más importantes pero menos inciertos son considerados invariantes estratégicas (en verde en el cuadro), mientras los menos importantes y menos inciertos son considerados variables de contexto (en amarillo en el cuadro). Esta forma de clasificar los factores críticos resulta una herramienta útil para poder discernir cuáles de estos, en sus posibles desdoblamientos (comportamientos contrapuestos), determinarán el futuro del sistema, indicando con ello las posibles ideas vertebradoras de los escenarios prospectivos. Así, mientras las invariantes estratégicas se visualizan como aspectos de alguna manera constantes en el desarrollo futuro, las incertidumbres críticas son las variables de peso en la visualización de distintos futuros posibles (futuribles).

A partir de este ejercicio se pudo comenzar a pensar en las ideas vertebradoras de los escenarios. Así, la primera conclusión fue que los factores críticos que más determinaban variaciones en el futuro del SAM eran los que hacen a la continuidad (o no) de las políticas públicas que inciden sobre este. Dejando de lado la escala internacional/regional (sobre la que se tiene mucha menor injerencia), los factores que aparecían como incertidumbres críticas eran tres de la dimensión político-institucional de la escala nacional/provincial/local (Proyecto de gobierno, Política industrial y agroindustrial, y Políticas de ordenamiento y gobernabilidad de los territorios), mientras en la escala focal, en la dimensión Socio-tecno-productiva, aparecían como incertidumbre crítica las políticas públicas específicas para la AUP. A partir de este resultado, se apreciaba que la continuidad del abordaje específico de la AUP en la región era lo más significativo para el futuro del SAM. Como expresamos an- teriormente, desde el INTA este abordaje había generado una institucionalidad acorde, algo que era acompañado también de políticas públicas de instituciones municipales, provinciales y nacionales (Barsky, 2013) ${ }^{15}$. El cambio de gobierno que aconteció a fines de 2015 parecía poner en cuestionamiento este proceso, lo cual fue vivido durante la realización del ejercicio como el principal condicionamiento a la continuidad del mismo $^{16}$. A partir de este resultado, se visualizó como idea vertebradora del devenir del futuro del SAM la discusión sobre el lugar de lo neo-público (Barsky, 2013) ${ }^{17}$, un concepto que da cuenta de esta nueva forma de abordar la AUP regional desde las políticas públicas.

Sin embargo, la segunda lectura que se desprendía del ejercicio de importancia-incertidumbre era la gran cantidad de factores invariantes, los cuales daban cuenta de procesos difíciles de modificar en su desenvolvimiento. A partir de estos, lo que se comenzó a dibujar fue la metáfora de la región metropolitana como un gran 'centro gravitatorio' que impone una fuerte 'inercia' sobre los procesos que en ella se dan. Por esto, más allá de la coyuntura política que tenía lugar, se imponía la idea de que tanto los procesos generales de la región (su crecimiento, sus problemas ambientales y sociales, su histórica falta de planeamiento, etc.), como los que hacían a la persistencia de las actividades productivas primarias en la misma, se proyectaban a futuro como aspectos 'inerciales' del propio proceso metropolitano, difíciles de modificar en sus aspectos fundamentales.

Esta forma de desenvolvimiento del SAM bo-

15 Como el Programa Nacional de Agricultura Periurbana iniciado en 2010, o institutos de desarrollo local como los creados en los partidos de Moreno, Pilar o Florencio Varela.

16 Durante la realización del ejercicio comenzó a aplicarse desde el entonces gobierno nacional una política de ajuste que ponía en disyuntiva la continuidad de las políticas públicas orientadas a la AUP regional.

17 Entendemos por neo-publico la revalorización de lo público, fortaleciendo las capacidades estatales, desde una perspectiva que revindica el valor de las culturas organizativas y la primacía de la política por sobre la autorregulación económica de la sociedad, sustentada a su vez por un rol activo de la ciudadanía en todos los niveles de la gestión pública (Barsky, 2013). 
naerense estaría inscripta en su heterogeneidad estructural, es decir, en la coexistencia de formas avanzadas en los sistemas de producción y consumo (de tipo capitalista) junto con formas de explotación de la fuerza de trabajo con características que podríamos denominar de pre-capitalistas (auto-explotación y precarización), las cuales siendo mutuamente funcionales hacen a la capacidad de estos sistemas de producción de readaptarse frente a situaciones de crisis o presión. Esta forma de desenvolvimiento del sistema fue caracterizada como su capacidad 'metamórfico/intersticial', ya que, si bien la presión por otros usos del suelo está siempre presente, los sistemas agroalimentarios de cercanía logran readaptarse, ya que del mismo modo la demanda urbana nunca cesa. Así, los mismos logran reubicarse en los intersticios de las zonas periurbanas, aunque a expensas de mayores y más ominosas formas de explotación y auto-explotación, así como de la pérdida de la calidad en los alimentos producidos y de mayores costos ambientales. Por todo esto y como uno de sus principales resultados, el ejercicio mostró cómo la persistencia de estas producciones es algo inherente al mismo funcionamiento inercial del sistema. Aclarando que dicha inercia no debe entenderse como una 'fuerza física', sino como una metáfora de la capacidad de los actores vinculados a la AUP metropolitana de sobreponerse a las adversidades, siendo agentes activos de su propio porvenir.

Tomando entonces como dimensión crítica la noción de desarrollo sostenible (en sus tres dimensiones, social, económica y ambiental), los escenarios futuros comenzaron a construirse a partir de estas dos ideas vertebradoras principales, la 'perspectiva neo-pública en cuestión' junto con la 'plasticidad inercial por atracción gravitacional' (Kozel et al., 2017). Con estas ideas se construyó un primer bosquejo de escenarios que fue puesto a consideración del grupo hacia fines de 2016. Frente a esto, y aun teniendo presente las dificultades por cambiar el rumbo de los procesos inerciales de región, el grupo discutió de qué manera se podían generar procesos disruptivos frente a estos escenarios tendenciales. Así, coincidiendo en que para el horizonte 2030 no se podrían vencer los procesos 'inerciales', se planteó un segundo horizonte a 2050 donde quizás sí se podrían desarrollar eventos disruptivos que lograsen torcer el desarrollo del SAM hacia dos escenarios disruptivos contrapuestos, basados en la 'dislocación' de la RMBA como centro 'gravitatorio' del país.

Con este esquema de escenarios, durante la segunda etapa del ejercicio los mismos se pusieron a consideración de más actores. Esta forma de trabajar en etapas y con miradas de distintos actores se debió al propio desenvolvimiento institucional de la experimental y del INTA en general, no a una decisión metodológica en sí misma. Así fue presentado ante el equipo de gestión ampliada de la experimental (integrado por técnicos e investigadores que no habían participado hasta entonces del ejercicio), y de su consejo asesor (integrado por un amplio grupo de representantes públicos y privados). En estas presentaciones, donde fue puesto en discusión la lógica y los resultados alcanzados hasta ese momento en el ejercicio, se llamó la atención sobre el sobredimensionamiento de la producción hortícola respecto a las demás formas de producción de la región. A partir de esto, se buscó caracterizar de mejor manera la gran diversidad productiva y su inherente multifuncionalidad. Así se realizaron talleres especiales sobre producción animal, junto con reuniones de trabajo con equipos de algunos de los territorios que no había podido relevarse en la primera etapa, como el territorio norte de la RMBA. Así se avanzó en una mejor caracterización del SAM en su diversidad, al mismo tiempo que los escenarios eran afianzados y reconfirmados en su lógica de desenvolvimiento.

\section{ESCENARIOS PROSPECTIVOS: LOS FUTUROS DEL SAM BONAERENSE}

El modelo de escenarios quedó definido por cuatro escenarios tendenciales, más dos disruptivos $^{18}$ (Cuadro 2). Los cuatro escenarios

18 En los ejercicios prospectivos es más común trabajar con no más de cuatro escenarios, donde solo se trabaja uno tendencial, junto con uno normativo, otro disruptivo y otro de- 
centrales dan cuenta del peso de los procesos 'inerciales', siendo dos de ellos, la continuidad del SAM bajo dos formas distintas de pensar la política pública. Una, como forma de acompañamiento y empoderamiento de los actores más involucrados en el SAM, denominado Neo-público; y otra basada más en la competitividad como forma de sostenimiento de las actividades productivas, denominado por ello Neo-empresarial. Junto a estos, otros dos escenarios se inscriben aún en

seado. Aquí, dadas las características del ejercicio antes expuestas, se optó por una forma nueva, que articulara las ideas vertebradoras que se construyeron colectivamente. una lógica de tipo inercial pero que aun así logran desplazarse en parte la misma, aunque con consecuencias diametralmente opuestas, denominados Sostenido hacia lo sostenible y Pendular insostenible.

A más largo plazo, mientras tanto, de forma disruptiva en un horizonte de tiempo más profundo (2050), se visualizaron dos escenarios donde la lógica de inercia (sobre el foco del ejercicio) es dislocada, es decir logra vencerse, aunque también bajo dos formas contrapuestas, denominados Reconfiguración territorial e Híper-concentración con conflictividad social.

Cuadro 2. Escenarios prospectivos del SAM al 2030/2050

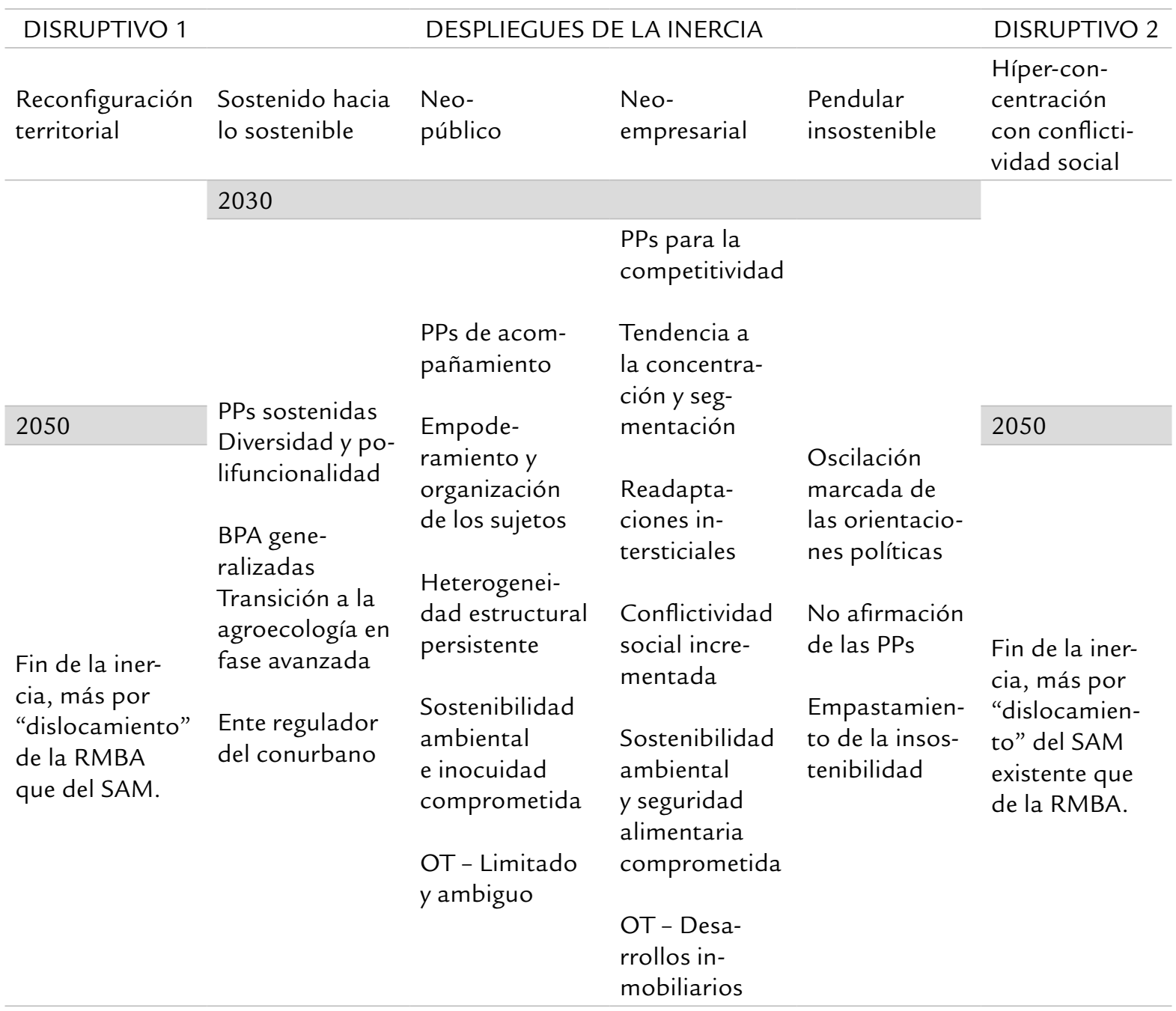

Fuente: elaboración personal 
Caracterizando más en detalle los dos escenarios centrales del cuadro (Neo-público y Neo-empresarial), buscaron dirimir en parte la dicotomía entre distintas orientaciones políticas que intentan, al menos teóricamente, avanzar hacia el horizonte del desarrollo sostenible. Representan escenarios tendenciales porque no logran modificar de forma significativa la 'inercia' del sistema, permaneciendo en ambos el alto contraste del SAM en relación a los conflictos por el uso del suelo, los problemas de seguridad alimentaria (tanto en términos de acceso como de innocuidad), los problemas ambientales, así como la heterogeneidad estructural de la región. La diferencia entre ambos radica en cómo se buscan atacar los problemas del SAM en relación a sus sistemas productivos de alimentos. En el escenario Neo-público, como sostuvimos, el objetivo es el acompañamiento y empoderamiento de los actores más vulnerables, como los vinculados a la agricultura familiar periurbana, con asistencia técnica y asociativa, centrada en tecnologías de procesos y en la búsqueda de salvaguardar los espacios productivos con ordenamientos territoriales acordes, para así apuntalar la seguridad alimentaria en la región con equidad social. Mientras tanto, en el escenario Neo-empresarial, el objetivo es lograr mayor competitividad de las explotaciones asociada a tecnologías de insumos que, junto con una política de control más estricta y ordenamientos territoriales más proclives al desarrollo inmobiliario, tienden a concentrar las actividades del SAM y con ello aumentando la conflictividad social y comprometiendo la seguridad alimentaria en la región.

Frente a estos dos escenarios centrales, se buscó construir otros dos que, aun insertos en la lógica inercial, se desplazaran de la misma hacia formas contrapuestas con componentes deseables e indeseables respectivamente. En primero de estos, denominado Sostenido hacia lo Sostenible, representa el escenario donde más se logra encauzar el desenvolvimiento del SAM desde los objetivos del desarrollo sostenible. Sin salirse del todo de la inercia sistémica, la continuidad y la consistencia en la orientación de las políticas públicas (tanto generales como focales), logran plasmar los objetivos del pensamiento planificador (lo que nunca había sido logrado hasta entonces). Así, muchos de los objetivos del escenario Neo-público son alcanzados y potenciados en el presente escenario. En esta imagen de futuro, la diversidad y multifuncionalidad del SAM es fuertemente valorada, se incorporan masivamente tecnologías de procesos, con la Agroecología como forma productiva primordial, se logran incorporar innovadoras formas de organización y comercialización que involucran a productores y consumidores $y$, si bien no se consiguen resolver por completo las problemáticas asociadas a la insostenibilidad sistémica (ambientales y sociales), sí se logran dar pasos importantes en este sentido. Con ello, las contradicciones entre discursos y prácticas que refieren al uso de agroquímicos son resueltas a través de un conjunto articulado de Buenas Prácticas Agrícolas (BPA) con predominio del paradigma agroecológico. En este escenario, el ordenamiento territorial prioriza firmemente los espacios verdes y productivos frente a la especulación inmobiliaria.

En contraposición, el escenario tendencial pero con componentes indeseados, denominado Pendular insostenible, representa la no afirmación de ninguna orientación política consistente. Con esto, la acumulación de problemas ambientales y sociales, en lo que podríamos llamar de 'empastamiento perdurable' de situaciones de insostenibilidad, llevan a la región como un todo a un eventual colapso sistémico. Tanto a nivel focal como en la dinámica general de la región, la alternancia de ciclos cortos entre las dos orientaciones políticas aludidas y las autolimitaciones producto de no dar continuidad a un abordaje integral de los problemas metropolitanos, llevan a déficits perennes de gobernabilidad hasta el punto de generar la anomia social generalizada. Sin haber profundizado en una mayor caracterización de las consecuencias de este escenario, se optó aun así por incorporarlo como un ejemplo de cómo la inercia sistémica, si no es reorientada, puede terminar de la peor 
forma como colapso social y ambiental.

Por último, se construyeron dos escenarios disruptivos para poder visualizar dos formas a más largo plazo donde la inercia sí logra vencerse (horizonte 2050), aunque con consecuencias diametralmente opuestas. Así, mientras en escenario disruptivo 1, con el nombre Reconfiguración territorial, es la propia dinámica expansiva de la RMBA la que logra contenerse producto de cambiar el 'centro gravitatorio' nacional, en el escenario disruptivo 2 , con el nombre de Híper-concentración con conflictividad social, es el propio SAM el que deja de funcionar como tal, desplazándose y concentrándose la producción de alimentos en otras regiones, y aumentando con ello la inseguridad alimentaria y la conflictividad social. Por ello, si bien en ambos la inercia sistémica es dislocada, esto se logra de maneras opuestas. En el primero esto se logra por una reconfiguración territorial de todo el país, donde la RMBA deja de ser la capital nacional, trasladándose a una ciudad mediterránea, perdiendo con esto la región gran parte de su poder 'gravitatorio'. Mientras tanto, en el escenario disruptivo 2, lo que se disloca es el funcionamiento del SAM como sistema de producción de alimentos de cercanía, lo que ha sido una de sus características históricas y estructurales. En este, producto de desplazar toda la producción de alimentos a zonas especializadas más distantes, incorporando paquetes tecnológicos de insumos e híper-concentrando la actividad, la seguridad alimentaria se ve gravemente comprometida al desarticularse el funcionamiento del SAM.

En el escenario disruptivo 1, lograr desplazar la capital nacional y su fuerza centrípeta trae como consecuencia disminuir enormemente la presión sobre el entorno periurbano, y con ella valorar y salvaguardar los espacios verdes y productivos. Realizando un escalonamiento con los procesos descriptos en el escenario tendencial Sostenido hacia lo sostenible, en lo que podría pensarse como una derivación disruptiva, el SAM resultante logra de forma mucho más contundente alcanzar los objetivos del desarro-
Ilo sostenible. Las producciones de cercanía son socialmente valoradas y su aporte a la resiliencia y sostenibilidad de la RMBA es, junto con el cambio de centro gravitatorio ya expuesto, la condición sine qua non para el sostenimiento de los millones de habitantes que todavía allí residen de formas social y ambientalmente justas.

El escenario disruptivo 2, mientras tanto, se basa en la idea de una forma mucho más concentrada de producción de alimentos (un poco a la manera del llamado Mar de Plástico en Almería, España). Aquí las disrupciones tecnológicas son las que permiten trasladar e híper-concentrar la producción en zonas especializadas distantes, transformando el ya aludido fenómeno de aumento de intensificación por incorporación del invernáculo, en el comienzo de un proceso mucho más profundo de cambio tecnológico. Sin embargo, dicho cambio no trae aparejadas mejoras en las condiciones alimentarias de la población en general, sino todo lo contrario. Dislocar el sistema agroalimentario metropolitano, haciendo que los alimentos frescos sean tratados cada vez más como commodities, hace que, si bien se abaraten los costos de producción, al ser comercializados cada vez más como mercancías insertas en lógicas de acumulación global, el costo de los mismos se encarezca cada vez más. De allí que este escenario disruptivo porte la problemática situación futura de conjugar menor disponibilidad con mayores problemas de acceso, aumentando la conflictividad social. A su vez, la híper-concentración logra desplazar a los actores más dinámicos del sistema, como lo era hasta entonces la agricultura familiar periurbana, sumando aún mayor conflictividad a todo el sistema metropolitano.

\section{CONCLUSIONES}

El siguiente ejercicio representa una forma exploratoria y los escenarios son más bien proto-escenarios que requerirían mayor profundización para construir escenarios completos. De todas formas, aun siendo exploratorio, un ejercicio de estas características brindó una nueva forma de practicar la prospectiva respondiendo 
rápidamente a los requerimientos institucionales. Los resultados en forma de escenarios representan una modelización teórica de cuáles pueden ser los futuros para el sistema agroalimentario metropolitano de Buenos Aires. No buscan acertar cuál es el futuro más probable. Su valor y utilidad residen en nutrir el debate actual sobre qué rol ocupa la producción agroalimentaria de cercanía en el sostenimiento y en la calidad de vida de millones de habitantes en la región. La manera en que se construyeron y la lógica en la que se basan intentan denotar la plasticidad de formas de producción de alimentos que son consustanciales con el desenvolvimiento de la metrópolis. Así, tanto la metáfora del 'poder gravitatorio' como la de 'plasticidad inercial' sirvieron para ilustrar la dinámica de este sistema agroalimentario. Los escenarios tendenciales representan la continuidad de las situaciones actuales mediadas por el rol de políticas públicas que, desde vertientes contrarias (Neo-público vs. Neo-empresarial), intentan orientar el desarrollo futuro del SAM. Los 'despliegues de la inercia' son entonces una manera accesible de representar el desenvolvimiento de un sistema sumamente complejo, lo mismo que los escenarios disruptivos son una forma de poder vencer esta lógica inercial. De allí que poder construir de forma colectiva estos escenarios, visualizando posibles futuros, resal-

\section{REFERENCIAS}

Aguirre, P. (2005). Estrategias de consumo: qué comen los argentinos que comen. Buenos Aires: Editorial Miño y Davila.

Allen, A. (2003). Environmental planning and management of the peri-urban interface: perspectives on an emerging field. Environment \& Urbanization, 15(1), 135-148.

Aubry, C.; Ramamonjisoab, J.; Dabatc, M.H.; Rakotoarisoad, J.; Rakotondraibee, J. y Rabeharisoaf, L. (2012). Urban agriculture and land use in cities: An approach with the multi-functionality and sustainability concepts in the case of Antananarivo (Madagascar). Land tando los factores que se piensan más cruciales para determinar el desenvolvimiento del SAM, representan instrumentos muy útiles para poder planificar y accionar desde el presente.

Como dijimos, por sus características, este ejercicio respondió de manera sui generis a un pedido institucional, en donde por los mismos motivos no se pudo evitar cierto sesgo 'intiano'. Aun así, entendemos, esto no fue en contra de la calidad del resultado final. Sin embargo, ejercicios prospectivos como el presente tienen sentido en tanto nutren debates que a la vez logran plasmarse en planes estratégicos concretos, con objetivos de más corto plazo que, como pequeños pasos en busca de una meta ulterior, logren ir orientando el desenvolvimiento presente hacia ese futuro deseado más distante. No existe otra forma de proyectarnos hacia el futuro que comenzar por problematizar el presente, y no existe presente que no se piense desde una idea de futuro que nos oriente. Este ejercicio de prospectiva territorial intentó iluminar los trayectos posibles. Los vaivenes internos que se han vivido en el INTA en los últimos años impidieron que se pudiera seguir profundizando en la lógica de los escenarios y en cómo éstos nutren la planificación estratégica de la institución. Aun así, resulta uno de los primeros intentos sistemáticos por pensar en el largo plazo para un sistema agroalimentario de primera importancia como lo es el de la RMBA.

Use Policy, 29, 429-439.

Aubry, C. y Kebir, L. (2013). Shortening food supply chains: A means for maintaining agriculture close to urban areas? The case of the French metropolitan area of Paris. Food Policy, 41, 85-93.

Bakker, N.; Dubbeling, M.; Gündel, S.; Sabel-Koschella, U.; y de Zeeuw, H. (2000). Growing Cities, Growing Food. Urban Agriculture on the Policy Agenda. Feldafing: Deutsche Stiftung für internationale Entwicklung (DSE) Zentralstelle für Ernáhrung und Landwirtschaft.

Barsky, A. (2005). El Periurbano Productivo, un 
espacio en constante transformación. Introducción al estado del debate, con referencias al caso de Buenos Aires. Scripta Nova, 9(194), s-n.

Barsky, A. (2007). La agricultura de "cercanías" a la ciudad y los ciclos del territorio periurbano. Reflexiones sobre el caso de la Región Metropolitana de Buenos Aires (pp. 15-30). En A. Svetlitza de Nemirovsky (Coord.) Globalización y agricultura periurbana en la Argentina. Escenarios, recorridos y problemas. Buenos Aires: FLACSO.

Barsky, A. (2013). Gestionando la diversidad del territorio periurbano desde la complejidad de las instituciones estatales. Implementación de políticas públicas para el sostenimiento de la agricultura en los bordes de la región metropolitana de Buenos Aires (2000-2013). Tesis doctoral. Departamento de Geografía, Facultad de Filosofía y Letras, Universitat Autònoma de Barcelona. Director: Antoni Francesc Tulla Pujol.

Barsky, A. y Aboitiz, P. (2012). Construyendo nueva institucionalidad en el INTA. La experiencia de creación de la agencia experimental AMBA (Área Metropolitana de Buenos Aires), especializada en agricultura periurbana. VII Jornadas de Sociología. San Miguel, Argentina.

Benencia, R. (2004). Trabajo y prejuicio. Violencia sobre inmigrantes bolivianos en la agricultura periférica de Buenos Aires. Revue européenne des migrations internationales, 20(1).

Benencia, R. y Quaranta, G. (2005) Producción, trabajo y nacionalidad: configuraciones territoriales de la producción hortícola del cinturón verde bonaerense. Revista Interdisciplinaria de Estudios Agrarios, (23), 101-132.

Boukharaeva, L.M. y Marloie, M. (2006). Family urban agriculture as a component of human sustainable development. CAB Reviews: Perspectives in Agriculture, Veterinary Science, Nutrition and Natural Resources, 1(025).

Colantoni, A.; Grigoriadis, E.; Sateriano, A.; Venanzoni, G. y Salvati, L. (2016). Cities as selective land predators? A lesson on urban growth, deregulated planning and sprawl containment. Science of the Total Environment, (545546), 329-339.
Cuellas, M.V. (2015). Control de la salinización del suelo mediante sistemas de drenes en producciones intensivas de cultivos bajo cubierta. Tesis de maestría, Facultad de Agronomía, Universidad de Buenos Aires.

Díaz Álvarez, C.J. (2014). Metabolismo urbano: herramienta para la sustentabilidad de las ciudades. Interdisciplina, 2(2), 51-70.

De Zeeuw, H.; Van Veenhuizen, R. y Dubbeling, M. (2011). The role of urban agriculture in building resilient cities in developing countries. Journal of Agricultural Science, (149), 153-163.

Dubois, A. (2018). Nurturing proximities in an emerging food landscape. Journal of Rural Studies, 57, 1-12.

FAO (2014). Growing Greener Cities in Latin America and the Caribbean. Roma: Food and Agriculture Organization of the United Nations (FAO).

Feito, M.C. (2017). Visibilización y valorización de la agricultura familiar periurbana. Intervenciones de políticas públicas en el partido de La Matanza. Mundo Agrario, 18(38), s-n.

Garay, A.M. (2003). Caracterización del sector Noroeste del AMBA (pp. 75-104). En A. Catenazzi y J.D. Lombardo (Comps.) La cuestión urbana en los noventa en la Región Metropolitana de Buenos Aires. Los Polvorines: Universidad Nacional General Sarmiento.

García, M. (2011). El cinturón hortícola platense: ahogándonos en un mar de plásticos. Un ensayo acerca de la tecnología, el ambiente y la política. Revista Theomai, (23).

García, M. y Le Gall, J. (2009). Reestructuraciones en la Horticultura del AMBA: tiempos de boliviano. IV Congreso Argentino y Latinoamericano de Antropología Social. Mar Del Plata, Argentina.

Giusti, M. y Prividera, G. (2015). Urbanización cerrada, agriculturización abierta (153-178). En S. Vidal-Koppmann (Comp.) Metrópolis en mutación. Buenos Aires: Editorial Café de las Ciudades.

Gorelik, A. (2015). Terra incognita: para una comprensión del Gran Buenos Aires como Gran Buenos Aires (tomo 6, pp. 21-69). En G. Kessler (Dir.) Historia de la Provincia de Buenos Aires. El Gran Buenos Aires. Buenos 
Aires: Unipe/Edhasa (dir. de la obra: Juan Manuel Palacio).

Grando, S.; Carey, J.; Hegger, E.; Jahrl, I. y Ortolani, L. (2018). Short Food Supply Chains in Urban Areas: Who Takes the Lead? Evidence from Three Cities across Europe. Urban Agriculture and Regional Food Systems, 2, s-n.

Guiomar, X. (2011). Les collectivités locales à la recherche d'une agriculture de proximité. Pour, 2(209-210), 169-183.

Instituto Nacional de Tecnología Agropecuaria INTA (2012). Agricultura Urbana y Periurbana en el Área Metropolitana de Buenos Aires: Creación de la Estación Experimental Agropecuaria AMBA. Buenos Aires, Ediciones INTA.

Jarosz, L. (2008). The city in the country: Growing alternative food networks in Metropolitan areas. Journal of Rural Studies, 24, 231-244.

Kozel, A.; Martínez, L.E.; Taraborrelli, D. y Carvalho, N. (2017). El sistema agroalimentario del Área Metropolitana de Buenos Aires. Ejercicio exploratorio de prospectiva territorial. Buenos Aires: Ediciones INTA.

Lipori, M.; Cad, M.; Palacios, D. y Barrionuevo, N. (2011). Agricultura familiar periurbana $y$ ordenamiento territorial en el Área Metropolitana de Buenos Aires. Un análisis diacrónico. GEOSIG, Revista Geografía y Sistemas de Información Geográfica, año 3(3), 244-261.

Medina Vásquez, J.; Becerra, S. y Castaño, P. (2014). Prospectiva y política pública para el cambio estructural en América Latina y el Caribe. Santiago de Chile: CEPAL.

Ministerio de Infraestructura, Vivienda y Servicios Públicos (2007). Lineamientos Estratégicos para la Región Metropolitana de Buenos Aires. Plan Estratégico Territorial. La Plata: Gobierno de la Provincia de Buenos Aires.

Miranda, M. (2017). Riesgos ambientales asociados al cultivo bajo cubierta en el cinturón hortícola del Gran La Plata. III Congreso Nacional de Ciencia y Tecnología Ambiental. Santa Fe, Argentina.

Morgan, K. (2009). Feeding the City: The Challenge of Urban Food Planning. International
Planning Studies, 14(4), 341-348.

Mougeot, L.J.A. (2000). Urban Agriculture: Definition, Presence, Potentials and Risks, and Policy ChaIlenges. Ottawa: International Development Research Centre (IDRC).

Opitz, I.; Berges, R.; Piorr, A. y Krikser, T. (2016). Contributing to food security in urban areas: differences between urban agriculture and peri-urban agriculture in the Global North. Agriculture Human Values, 33, 341-358.

Palacios-Argüello, L.; Morganti, E. y Gonzalez-Feliu, J. (2017) Food hub: Una alternativa para alimentar las ciudades de manera sostenible. Revista Transporte y Territorio, (17), 10-33.

Parés, G. (2009). Las funciones de la agricultura urbana y periurbana en La Matanza, desde la óptica de los propios agricultores, en la primera década del siglo XXI. Tesis de maestría inédita. Universidad Nacional de San Martín. Universidad Autónoma de Madrid. Buenos Aires. Madrid.

Pengue, W.A. (2017). Agroecología y Urbanismo en el siglo XXI: Hacia la generación de Escudos Verdes Productivos en los Pueblos y Ciudades de la Argentina. Revista Fronteras, año 15(15), 30-45.

Reed, M.; Mettepenningen, E.; Swagemakers, P.; Dominguez Garcia, M.D.; Jahrl, I. y Koopmans, M.E. (2018). The Challenges of Governing Urban Food Production across Four European City-Regions: Identity, Sustainability and Governance. Urban Agriculture and Regional Food Systems, 2, s-n.

Sali, G.; Corsi, S.; Mazzocchi, C.; Monaco, F.; Wascher, D.; van Eupen, M. y Zasada, I. (2014). FOODMETRES D2.1: FoodMetres Analysis of food demand and supply in the Metropolitan Region. Wageningen: Wageningen Environmental Research Institute (Alterra).

Satterthwaite, D.; McGranahan, G. y Tacoli, C. (2010). Urbanization and its implications for food and farming. Philosophical Transactions of the Royal Society B, (365), 2809-2820.

Smit, J. y Nasr, J. (1992). Urban agriculture for sustainable cities: using waste and idle land and water bodies as resources. Environment and Urbanization, 4(2), 141-152. 
Smit, J.; Ratta, A. y Nasr, J. (2001). Urban agriculture: Food, jobs and sustainable cities. New York, N.Y: United Nations Development Programme.

Sommers, P. y Smit, J. (1994). Promoting Urban Agriculture: A Strategy Framework for Planners in North America, Europe, and Asia. Ottawa: The Urban Agriculture Network.

Stupino, S.A.; Frangi, J.L. y Sarandón, S.J. (2012). Caracterización de fincas hortícolas según el manejo de los cultivos, La Plata, Argentina. VII Congreso de Medio Ambiente. AUGM, UNLP, La Plata.

Tacoli, C. (1998). Rural-urban interactions: a guide to the literature. Environment and Urbanization, 10(1), 148-166.

van Veenhuizen, R. (2006). Cities Farming for the Future: Urban Agriculture for Green and Productive
Cities. Leusden: RUAF Foundation, IDRC and IIRR Publishing.

van Veenhuizen, R. y Danso, G. (2007). Profitability and sustainability of urban and peri-urban agriculture. Roma: FAO.

Verdaguer Viana-Cárdenas, C. (2010). La agricultura periurbana como factor de sostenibilidad urbano-territorial. Conclusiones preliminares del estudio de casos desde la perspectiva del planeamiento urbanístico (pp. 7-29). En M. Vázquez y C. Verdaguer (Dirs.) El espacio agrícola entre el campo y la ciudad. Madrid: Edición Convenio Ayuntamiento de Vitoria-Gasteiz y Universidad Politécnica de Madrid.

Vitale, J.; Pascale Medina, C.; Barrientos, M.J. y Papagno, S. (2016). Guía de prospectiva para el ordenamiento territorial rural de la Argentina a nivel municipal. Buenos Aires: INTA Ediciones.

\footnotetext{
Lisandro Esteban Martínez es Licenciado en Antropología de la Universidad Nacional de Rosario (2010) y Diplomado en Estudios Avanzados en Evaluación de Políticas Públicas de la Universidad Nacional de General San Martín (2014). Es Investigador del Centro de Investigación en Economía y Prospectiva (CIEP). Se especializa en el estudio de los sistemas alimentarios metropolitanos, con especial atención a las producciones de cercanía. Ha participado, coordinado y organizado diversos talleres, congresos y seminarios, así como publicado diversos trabajos de investigación, artículos y libros. Ha sido coordinador del ejercicio de prospectiva territorial sobre el Sistema agroalimentario de la RMBA al 2030/2050. Instituto Nacional de Tecnología Agropecuaria (INTA). Av. Cerviño 3101 P.B. (C.P. 1425) Ciudad de Buenos Aires, Buenos Aires, Argentina, martinez.lisandroe@inta.gob.ar
} 